Retos, 9(I8), 2019

\title{
Happiness Management en la época de la Industria 4.0
}

\section{Happiness Management in the age of Industry 4.0}

Dr. Rafael Ravina-Ripoll es profesor e investigador de la Universidad de Cádiz (España) (rafael.ravina@uca.es) (https://orcid.org/0000-0001-7007-3123)

Dr. José Marchena Domínguez es profesor e investigador de la Universidad de Cádiz (España) (jose.marchena@uca.es) (https://orcid.org/0000-0002-7612-7484)

Dr. Miguel Ángel Montañés-Del Río es profesor e investigador de la Universidad de Cádiz (España) (miguelangel.montanes@uca.es) (https://orcid.org/0000-0001-6032-9965)

\section{Resumen}

La era de la industria 4.0 se está caracterizando, por un lado, en la existencia de sistemas de gobernanza que gradualmente está erosionando el estado de bienestar, y por otro, en la implementación de modelos de gestión empresariales basados en la precariedad laboral y la reducción masiva de puestos de trabajos derivados de la automatización de los procesos productivos y el uso extensivo de los robots. Todos estos factores influyen negativamente en la felicidad de los seres humanos, especialmente en ecosistemas que viven alejados de los principios rectores del bienestar y la justicia. Ante esta realidad, el objeto de este artículo es discutir, a partir de una revisión crítica de literatura académica, que el «Happiness Management» constituye una importante pieza axial en la generación de empresas que cultiven el círculo virtuoso de la felicidad y la creación de una atmósfera positiva en el interior de las organizaciones que estimulen la innovación y la creatividad. De esta forma se puede construir -en plena perturbación tecnológica-, un capitalismo inclusivo que amortigüe las desigualdades sociales a partir de empresas que lleven a cabo modelos de gestión sustentados en el «Happiness Management», teniendo presente que el capital humano del sector industrial no está aprovechando los frutos que originan los robots en su trabajo diario. Esto también precisa efectuar una breve reflexión sobre modelos de sostenibilidad medioambiental y convivencia social que garanticen un futuro generacional estable y feliz.

\begin{abstract}
The era of Industry 4.0 is characterized, on the one hand, by the existence of governance systems that are gradually eroding the Welfare State, and on the other hand, by the implementation of business management models based on precarious work and the massive reduction of jobs. This is a result of the automation of production processes and the widespread use of robots and automation. All these factors negatively influence human happiness, especially in ecosystems that live far from the guiding principles of wellbeing and justice. Faced with this reality, the purpose of this article is to indicate that the Happiness Management constitutes an important component in the creation of companies that cultivate a virtuous circle of happiness and the creation of a positive atmosphere inside organizations. These qualities stimulate innovation and creativity and help to build an inclusive capitalism, in the midst of technological perturbation. They cushion companies that employ management models based on Happiness Management from social inequalities. We must take into account that the human capital of the industrial sector is not taking advantage of the benefits provided by robots in their daily work. This investigation will also require a brief reflection on models of environmental sustainability and social coexistence that guarantee a stable and happy future generation.
\end{abstract}

\section{Palabras clave I keywords}

Felicidad, industria 4.0, sostenibilidad, innovación, bienestar, recursos humanos, creatividad, empresa. Happiness, industry 4.0, sustainability. innovation, wellbeing, human resources, creativity, firm.

Cómo citar: Ravina-Ripoll, R., Marchena Domínguez, J., y Montañés-Del Río, M.A. (2019). Happiness Management en la época de la Industria 4.0. Retos Revista de Ciencias de la Administración y Economía, 9(18), 189-202. https://doi.org/10.17163/ret.n18.2019.01. 


\section{Introducción}

La felicidad y la consecución del bienestar fue un objetivo identificable que movió históricamente a individuos y colectivos sociales y que, hoy en día, sigue siendo leitmotiv de las políticas, la economía y el pensamiento. Un concepto transversal pero que precisa su definición y secuenciación para entender mejor muchas de las claves que caracterizan la era de la Industria 4.0. Una época que va a traer consigo nuevos modelos de producción y de gestión de los recursos humanos (Popkova, Ragulina \& Bogoviz, 2019). Desde esta vertiente, las empresas deberán, por un lado, tener estructuras flexibles y dinámicas que permitan dar respuesta rápidamente a las exigentes demandas del mercado (Lasi, Fettke, Kemper, Feld \& Hoffmann, 2014); y por otro lado, poseer una fuerza laboral no solo con una alta formación académica y profesional, sino también con una mentalidad abierta hacia la innovación, el pensamiento creativo, las habilidades sociales, el trabajo en equipo, la resolución de problemas, entre otros (Benešová \& Tupa, 2017; Romero-Rodríguez \& Castillo-Abdul, 2019). Esto precisará, entre otras cosas, de un modelo de gestión o una cultura corporativa donde se vincule directamente la felicidad del capital humano con aspectos como producción, eficiencia y competitividad. El cultivo de este constructo, junto a la búsqueda integral de la satisfacción del cliente hiperconectado, estimulará significativamente la rentabilidad económica y financiera de las grandes multinacionales, así como de las pequeñas y medianas empresas (Baker, Greenberg \& Hemingway, 2006).

A mediados de 2016 Smit, Kreutzer, Moeller y Carlber elaboran un estudio para la Unión Europea ${ }^{1}$ en el que demuestran cómo la Industria 4.0 traerá a la economía de este territorio un semillero de entidades productivas que se verán forzadas a implantar modelos flexibles de negocios. De esta manera, dichas compañías podrán ofrecer bienes o servicios de calidad a sus clientes potenciales. Ello requiere, por un lado, que las empresas se adapten rápidamente a los cambios tecnológicos, y por otro, que gocen de una cultura organizativa que permita mejorar el bienestar colectivo de los empleados en la sociedad digital. Bajo nuestro punto de vista, esto último se podría cosechar cuando la alta dirección de las empresas empezase a interiorizar que la gestión de la felicidad o «Happiness Management», ayuda a mitigar el pensamiento económico de Milton Friedman, inspirado básicamente en que el único objetivo de las corporaciones deben ser la maximización del beneficio monetario de sus accionistas. Con el objeto de confrontar esta idea en las próximas páginas se sugiere, grosso modo, que el «Happiness Management» constituye un modelo de gestión que brinda la posibilidad de incentivar la felicidad colectiva a través de los siguientes vectores: la flexibilidad, el optimismo, la confianza, el compromiso, la confianza y el aprendizaje (Vázquez, 2012).

\section{Revisión de literatura y estado de la cuestión}

A lo largo de los últimos lustros del siglo XXI han surgido en el mundo científico una pléyade de monográficos sobre la moderna disciplina académica de la economía

1 Disponible en línea en: https://bit.ly/1QRykFi (Fecha de consulta: 20/03/2019). 
de la felicidad (Frey \& Stutzer, 2018; Sánchez-Vázquez, 2018; Crespo \& Mesurado, 2015; Rodríguez-Muñoz \& Sanz-Vergel, 2013; Nelson, 2010; Kahneman \& Krueger, 2006). Este fenómeno cognitivo puede venir originado fundamentalmente por tres factores: El primero de ellos es que a principios del presente siglo, concretamente en 2003, Hills y Argyle elaboran el cuestionario de la felicidad de Oxford (OHQ) ${ }^{2}$. Este instrumento psicométrico sirvió para demostrar que los seres humanos que disfrutan en su vida cotidiana de una alta tasa de felicidad es básicamente fruto del desarrollo de las siguientes variables: extroversión, amabilidad, sentido del humor, sentido de propósito, apreciación estética, autonomía, autoeficiencia, salud física, y autoestima (Arunachalam, 2018). Desde ese momento un volumen importante de especialistas de este tópico académico ha comenzado a usar de forma generalizada dicho cuestionario en sus estudios empíricos sobre los constructos compromiso organizacional-felicidad laboral y liderazgo- felicidad en la era de la industria 4.0 (Salas-Vallina, López-Cabrales, Alegre \& Fernández, 2017; Wang \& Yang, 2016).

El segundo de los factores aludidos hace referencia a que, durante las últimas décadas, la mayoría de la producción científico-académica sobre la felicidad tiene en común el uso en sus investigaciones del propio término «felicidad» como sinónimo de bienestar subjetivo, de satisfacción laboral o de calidad de vida (Seligman, 2016; Mackerron, 2012: Oishi, 2012, Rojas, 2009; Zelenski, Murphy \& Jenkins, 2008; Diener, 2000). Tal hecho no estuvo exento de sus correspondientes debates académicos. Sin embargo, dicho axioma científico sirvió, entre otras cosas, para atraer la atención de economistas, sociólogos y psicólogos en analizar estadísticamente si la felicidad de los seres humanos presenta una correlación significativa con las variables independientes: edad, sexo, religión, estado civil, salud o nivel de estudios (Graham \& Pozuelo, 2017; Zweig, 2015; Cuñado \& De Gracia, 2012; Frijters \& Beatton, 2012; Andersson, 2008; Lu, 2000). En este sentido, se hace conveniente poner de manifiesto que en la actualidad muchos de los resultados de estos trabajos suelen ser criticados académicamente ya que miden o bareman la felicidad de forma subjetiva, y, por ende, no de forma empírico-científica (Ahn \& Mochón, 2010). Dicha aseveración se debe a que por regla general usan como principal fuente de información primaria las respuestas que facilitan los individuos cuando se les preguntan en las encuestas: ¿en qué medida se considera Ud. una persona feliz o infeliz? Este ítem se encuentra no solo en la encuesta que elabora anualmente la consultora estadounidense Gallup a escala internacional sobre la felicidad, sino también en los barómetros que efectúa el Centro de Investigaciones Sociológicas (CIS) en España desde 2013. Esto mismo no ocurre con el informe anual que diseña la Organización de Naciones Unidas (ONU) sobre el ranking mundial de los países más felices del mundo, pues mide la felicidad a partir del estudio de las siguientes ratios: Producto Interior Bruto, ayudas sociales, esperanza de vida, libertad, percepción de la generosidad, corrupción y calidad de vida de los inmigrantes. Teniendo en cuenta esta diferencia metodológica, no es de extrañar que

2 Disponible en línea en: https://bit.ly/2YsZrRI (Fecha de consulta: 21/03/2019). 
se observen fuertes discrepancias en la clasificación de felicidad ofrecida por Gallup y la ONU para el año $2017^{3}$.

Y el tercer factor no se puede entender sino se hace referencia a que, a mediados de 2011, la ONU emite una resolución donde se afirma que "la búsqueda de la felicidad es un objetivo humano fundamental y encarna el espíritu de los objetivos acordados a nivel mundial conocidos como los Objetivos de Desarrollo del Milenio" (ONU, 2011). Estas palabras se pronuncian en plena ebullición de una extensa y amplia literatura científica sobre la economía de la felicidad, así como en medio del desarrollo de múltiples eventos científicos sobre este tópico en las instituciones de enseñanza superior de Europa y América (Rojas, 2016). Tal hecho alimentó el apetito intelectual para explorar los estudios de la polisemia de la felicidad bajo el prisma de la multidisciplinaridad y la transversalidad académica. Desde este enfoque se puede refutar al mismo León Tolstói cuando escribió en Guerra y Paz que la felicidad es monótona y uniforme (Vázquez \& Hervás, 2009). Hoy por hoy, se sabe que la felicidad colectiva viene determinada por múltiples vectores, entre ellos, la existencia de territorios que promuevan el progreso social de los ciudadanos mediante el desarrollo de una economía circular, innovadora y ecológica (Villena-Manzanares \& GalianoCoronil, 2017). Ello facilita la construcción de un estado de bienestar bajo las sinergias de la sostenibilidad, el diálogo social, la competitividad, el emprendimiento y la inversión pública (Ravina-Ripoll, Tobar-Pesantez \& Galiano-Coronil, 2018; Rothstein, 2010; Pacek \& Radclif, 2008).

Toda vez ofrecida en las líneas anteriores una diáfana imagen sobre la producción bibliográfica de la economía de la felicidad como una disciplina que se encuadra dentro de la economía del bienestar (Esteve, 2004), se hace conveniente advertir la existencia de un escaso número de investigaciones científicas destinadas a estudiar empíricamente el término "Happiness Management» en la época de la Industria 4.0 (Nogueira et al., 2017; Villegas, et al., 2015; Cera, 2012). Quizás este hecho venga derivado, por un lado, a consecuencia de que cuando alguien lee esta atractiva expresión, la asocia directamente con la expresión felicidad corporativa o laboral (Pryce-Jones \& Lindsay, 2014). En este sentido, Salas-Vallina, Alegre y Guerrero (2018) revelan que los máximos responsables de las organizaciones deben apostar por un modelo de gestión que incentive la felicidad laboral mediante el compromiso individual, la satisfacción laboral, el liderazgo transformacional y el altruismo; y por otro lado a la ausencia de una clara conceptualización de la palabra felicidad en el área académica de organización de empresa, atendiendo a la documentación consultada para la elaboración de este artículo. Ante esta realidad, se hace preciso fomentar grupos de investigación que aborden el estudio del «Happiness Management» como una rama de la ciencia de la felicidad que aflora con la misión de enseñar holísticamente la «Felisofía». Por esta acepción se entiende la cultura o la filosofía organizacional que permite a los dirigentes de las administraciones públicas y las empresas propulsar políticas estratégicas integrales donde la búsqueda de la felicidad -tanto individual como corporativa-, esté por encima de los intereses de las grandes élites (Ravina-

3 En ese año las Islas Fiji fue el país más feliz del mundo para Gallup, mientras que para la ONU fue Noruega (La Vanguardia, 2018; World Happiness Report, 2017). 
Ripoll, 2017). Ello ayudará a impulsar la construcción de un mar de ecosistemas y tejidos productivos más innovadores, emprendedores, sostenibles, ecológicos y creativos en la sociedad digital (Ravina-Ripoll,Tobar-Pesantez \& Núñez-Barriopedro, 2019; Dolan \& Metcalfe, 2012).

La propiedad intelectual del término «Felisofía» ${ }^{4}$ emerge dentro del grupo de investigación IGOMSOH (Iberoamerican Group of Multidisciplanary Studies on Happiness) de la Universidad Politécnica Salesiana (Ecuador). Entre sus principales objetivos de investigación se encuentra, por un lado, seguir profundizando en el análisis del «Happiness Management» como un instrumento válido y eficaz para el diseño de estrategias innovadoras de dirección organizacional en la época de la Industria 4.0 (Ravina-Ripoll, Villena-Manzanares \& Gutiérrez-Montoya,2017); y por otro lado, intensificar la producción científica sobre el estudio de la felicidad desde un enfoque transdisciplinar y transversal (Ravina-Ripoll, et al., 2019; Ravina-Ripoll, TobarPesantez \& Marchena-Domínguez, 2019). Esta rica información puede ser muy interesante para explorar cómo influyen el bienestar subjetivo y la felicidad corporativa en el desarrollo sostenible y medioambiental de los estados de bienestar en la actual sociedad digital (Frey \& Stutzer, 2010; Rego, Ribeiro \& Cunha, 2010).

Recapitulando, y a pesar del poco espacio de tiempo transcurrido desde la emisión del cuestionario de la felicidad de Oxford (OHQ, op. cit), algo más de tres lustros han permitido fraguar un concepto que comienza a aplicarse a la vertiente laboral desde pautas organizativas y de liderazgo, con el objeto de compensar los desajustes provenientes de la era de la Industria 4.0. Un estudio de la felicidad que, ya sea de manera subjetiva o empírica, nos aporta una información precisa para abordar los parámetros más interesantes y culminar la felicidad como un valor humano fundamental, desde la gestión de las organizaciones implicadas y desde el estudio de especialista y proyectos que le dan fuste y contenido (Ferrer \& Carbonell, 2013; Blanchflower \& Oswald, 2011; Pacek \& Radcliff, 2008).

\section{Materiales y método}

El principal objetivo del presente trabajo es coadyuvar en la comprensión sobre el moderno concepto del "Happiness Management» contextualizado en la era de la Industria 4.0. Para ello se ha realizado un análisis bibliométrico y hermenéutico de tipo generalista (Will, Bertrand \& Fransoo, 2002; Wacker, 1998), lo que puede contribuir significativamente a mejorar el conocimiento de esta disciplina académica y por ende, del estado de la cuestión sobre este atractivo y novedoso tópico, lo que puede resultar muy interesante para que surjan futuros estudios multidisciplinares sobre esta área empresarial de la economía de la felicidad.

4 Este concepto nace con el objeto de poner en el centro de gravedad de los responsables de las administraciones públicas y las empresas que el desarrollo sostenible de los territorios debe proceder de la puesta en marcha de políticas estratégicas integrales donde la búsqueda de la felicidad holística de los ciudadanos está por encima de la economía y los intereses particulares de las grandes élites. De esta manera se podrán tener un tejido productivo más inclusivo, saludable, tolerante, etc. A modo de curiosidad, indicar que la propiedad intelectual de Felisofía está registrada en el Ministerio de Educación, Cultura y Deporte de España con el número 14/2018/248. 
Respecto a la elección de la franja temporal, se optó por elegir el intervalo de tiempo comprendido desde el año 2000 hasta la actualidad (2019), debido fundamentalmente a la novedad incipiente de este término en el mundo científico como se leerá más adelante. Tras esto, se decidió emplear como filtro de elección la palabra «Happiness Management» con el algoritmo booleano AND y la selectividad por los metadatos «tema» (que incluye título, resumen y palabras clave). Los motores de búsqueda seleccionados fueron las plataformas Scopus y la Web of Sciences (Wos). La consulta originó una muestra representativa de menos de diez artículos indexados. A modo de curiosidad indicar que el primer texto donde figura este término en el título de un artículo se escribió en 2017 por Nogueira Kamel, Martins, Pessanha y De Andrade en la revista Brazilian Journal of Science and Technology.

Seguidamente se aplicó un segundo filtro, que consistió en buscar en estas dos fuentes de información primaria el concepto de «Happiness Management» sin entrecomillar. Ello se tradujo en casi veinte artículos, la mayoría de ellos pertenecientes a la disciplina del Business y el Management. Entre los múltiples factores que explican este fenómeno, se encuentra quizás el gran interés que tienen los máximos responsables de la dirección estratégica de los recursos humanos en crear una cultura de felicidad en el interior de sus organizaciones (Blackman, O'Flynn \& Mishra, 2010).

Ahora bien, ninguno de los trabajos incluidos en las bases de datos Scopus y Wos contempla una definición del vocablo «Happiness Management», lo que dificulta sin lugar a duda seguir avanzado en el desarrollo exponencial sobre su estudio. Esto puede ser muy útil para construir una sociedad más humanista y abierta al interés general de la ciudadanía. De ahí que en el siguiente apartado se proponga una interpretación sobre este tópico, así como una definición del término felicidad corporativa.

\section{Happiness Management: Un modelo de gestión multicultural a descubrir en la sociedad digital}

La era de la industria 4.0 se caracteriza por la automatización de los procesos productivos que genera la ciberfísica, la computación, la robótica y la ingeniería. No es poco sostener que la incorporación de todos estos avances tecnológicos en la vida cotidiana de las empresas supondrá una fuerte reducción en sus costes de fabricación, y por ende, un mayor nivel de competitividad y sostenibilidad en la época de la sociedad digital (Saucedo-Martínez, et al., 2018; Moreno et al., 2017; Prause \& Weigand, 2016; Maynard, 2015; Sommer, 2015). Dicha realidad parece olvidar el viejo adagio que decía que uno de los activos más importantes que poseen las compañías es el talento creativo de su capital humano. De ahí quizás la existencia de muchas empresas carentes de una cultura destinada a fomentar un clima organizacional bajo los principios rectores de la motivación, el trabajo colaborativo, la fidelización, el compromiso, la responsabilidad social y el bienestar laboral.

Aunque los vectores causantes de dicho fenómeno no son objeto de estudio de la presente investigación, sí se intentará explicar, grosso modo, el moderno concepto del «Happiness Management». Como se ha dicho anteriormente, si se busca esta 
expresión entrecomillada en las bases de datos Scopus y WoS se detecta que solo se han escrito cinco artículos desde 2009. Todos ellos tienen en común que entienden el «Happiness Management» como un instrumento válido y eficaz para vitalizar la satisfacción laboral de sus empleados, mientras que no ofrecen un concepto o definición del citado término en relación con la era de la Industria 4.0. Esto nos lleva a delimitar, desde un enfoque axiológico-conceptual la construcción de una primera definición de «Happiness Management»: Modelo de gestión multicultural destinado a incentivar en el desempeño del puesto de trabajo los siguientes recursos: creatividad, compromiso, innovación tecnológica, emprendimiento interno y responsabilidad social. De esta manera se podrán impulsar en el interior de las organizaciones el virtuoso círculo de la felicidad corporativa.

\section{Figura 1. Factores existenciales del «Happiness Management»}

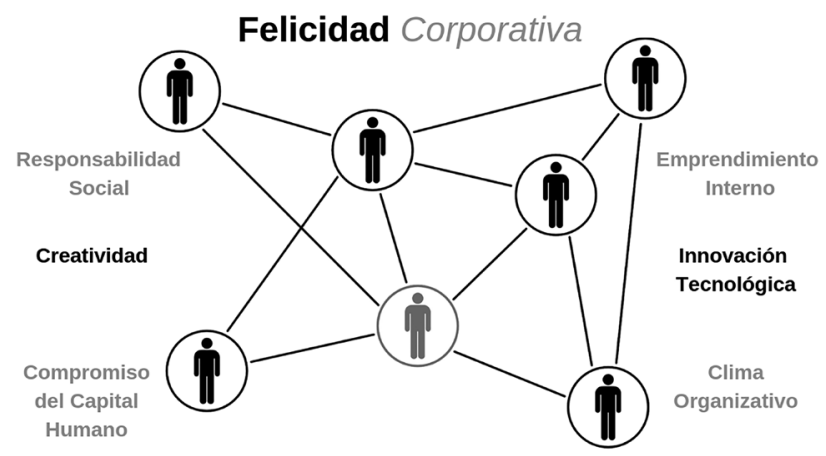

En la Figura 1 se observa que aparece la palabra «felicidad corporativa» sin que en la actualidad exista ninguna conceptualización sobre este término, según se desprende de la consulta para el desarrollo de la presente investigación. Tal hecho ofrece la posibilidad de describir la felicidad corporativa como la tasa media de bienestar subjetivo o, de felicidad individual que poseen los clientes internos de una organización en el desempeño diario de sus puestos de trabajo durante un determinado intervalo de tiempo (Ahumada-Tello et al., 2018).

Desde este apriorismo, se hace conveniente apuntar que la felicidad corporativa no puede interpretarse como la mera suma agregada de la felicidad laboral y organizacional, pues su implementación precisa de la implicación activa de la cúpula directiva y de la totalidad de los clientes internos. Por tanto, la felicidad organizacional se debe entender como el compromiso que tiene la alta dirección por acrecentar la felicidad corporativa mediante la generación de un clima laboral que esté abierto hacia la innovación tecnológica, el lenguaje constructivo, la interacción, la comunicación entre iguales, etc. Así se podrá cultivar un activo intangible que estimule las emociones positivas, la creatividad y las relaciones interpersonales, entre otras (Núñez-Barriopedro, Ravina-Ripoll \& Tobar-Pesántez, 2019). 
En este sentido, no es extrañar que las grandes multinacionales del mundo globalizado ponga en marcha acciones destinadas a incrementar exponencialmente la motivación laboral y el bienestar psicosocial de sus recursos humanos, sirvan de ejemplo Google, Toyota, KPMG, IKEA, Facebook o Linkedin (Equipo \& Talento, 2018).

De acuerdo con lo anterior, y teniendo presente que cada individuo tiene su propia percepción de felicidad subjetiva, ya se podría definir el término «felicidad laboral» en la sociedad digital. El presente concepto está originando un gran debate en el mundo académico, pues muchos estudiosos entienden que la felicidad laboral está determinada únicamente por la satisfacción o el placer positivo que consiguen los empleados a corto plazo en el mero cumplimiento de sus tareas funcionales (Bandura y Lions,2014). Otros investigadores consideran que la felicidad laboral de las organizaciones viene explicada por la satisfacción laboral y otras múltiples variables, sirva de ejemplo, el engagement, la implicación laboral, el estilo de liderazgo, la confianza, el compromiso, la confianza, el puesto de trabajo, la cultura orientada al trabajo en equipo, el desarrollo organizacional, la gestión del conocimiento o la política de recursos humanos (Oswald, Proto \& Sgroi, 2015; Yuan, 2015; Fisher, 2010).

Así, cabría preguntarse ¿cómo lograr una organización feliz en la era de la Industria 4.0? Quizá la respuesta se encuentre en el «Happiness Management», un modelo de gestión que es capaz de generar una cultura innovadora cimentada en la construcción del círculo virtuoso de la felicidad corporativa, donde los clientes internos tengan cada día más ilusión por ir a trabajar y ser más productivos. Teniendo presente que los pequeños incrementos que se produzcan en la felicidad colectiva de las organizaciones darán lugar a aumentos en una proporción bastante superior sobre los rendimientos económicos o sociales.

En esta línea de reflexión habría que dejar patente dos cosas: Por un lado, la significativa ausencia del «Happiness Management» en la dirección estratégica de las organizaciones, y por el otro, la escasez de publicaciones encaminadas a investigar al «Happiness Management» como una herramienta muy interesante para conseguir un océano lleno de empresas innovadoras que graviten en torno a la felicidad de su capital humano y no a la maximización de las ganancias económicas y financieras (Nogueira et al., 2017).

\section{Discusión y conclusiones}

La felicidad es un concepto de general identificación y al que probablemente todo individuo quiera aspirar, pero que entraña dificultades a la hora de definir sus perfiles, valorar sus claves y enumerar sus recetas para lograrla. La felicidad ha sido un hecho perceptible desde siempre. Ahora, sin embargo, y desde la teorización de su conocimiento, parece obvio considerarla como un bien tangible y un reto indispensable para garantizar el futuro y la prosperidad de los seres humanos. Basándose en esta última idea, parece clara la necesidad de construir y relacionar estudios que diseccionen, descompongan y extraigan las partes principales de sus claves. Luego, identificarlas y conocerlas y, finalmente, establecer modelos y herramientas que posibiliten diagnosticar y garantizar tan indispensable valor social y colectivo. 
Existen estudios que van construyendo diversas líneas de ensamblaje de la felicidad, pero no son aún suficientes. Es precisa una visión de conjunto y aspirar a identificar la felicidad -como coincide la mayoría de estos trabajos- como un modelo transversal que interesa aspectos políticos, económicos y culturales. Una realidad determinada por variables físicas, psíquicas y potenciales; por valores empíricos, pero también subjetivos; por estrategias y planes de diseño. No en balde, las sociedades modernizadas auspiciadas por el welfare state intentaron cubrir las necesidades primarias, encabezadas principalmente para el logro de la felicidad. De la misma forma, la economía precisaba de ser un modelo eficaz e intenso que fuera capaz de superar los altibajos y las consecuencias indeseables.

En este sentido se apuntan las alternativas microeconómicas o el «Happiness Management» como un atractivo modelo de gestión que frene la excesiva tecnificación en la era de la Industria 4.0 desde la asunción de responsabilidades por parte de las instancias directivas que cuiden del talento y del capital humano. Para ello las organizaciones deben cultivar básicamente dos aspectos: El primero, incentivar una cultura empresarial que considere a sus empleados como una fuerza motora de innovación, creatividad o trabajo en equipo; y el segundo, implementar el modelo del «Happiness Management» como una pieza axial que juega un papel muy relevante para que los máximos responsables de las compañías contemplen a sus trabajadores más allá del viejo rol del homo económicus.

A partir de estos dos vectores, se pueden construir empresas que contemplen la búsqueda de la felicidad corporativa como el medio para maximizar el rendimiento financiero y lograr ventajas competitivas de naturaleza sostenible y duradera en el tiempo. De ahí la necesidad de seguir investigando en el desarrollo del término «Happiness Management», con la finalidad de demostrar que la viabilidad económica de las organizaciones se encuentra en impulsar holísticamente la felicidad colectiva de sus clientes internos, y poseer un volumen importante líderes y mandos intermedios más empáticos, sociales y altruistas con el desempeño de su puesto de trabajo.

Antes de finalizar indicar, por un lado, que a lo largo del presente texto se ha mostrado de forma generalista la relevancia que puede tener la aplicación del «Happiness Management» en la era de la Industria 4.0. Ahora bien, existen una serie de limitaciones que se deberán ir superado con la elaboración de futuras producciones científicas. Entre ellas cabe citar la ausencia de una definición académica de este tópico, lo cual ayudaría a elaborar estudios de carácter empíricos y metodológicos con el objeto de poder explorar la fuerte implicación que posee el «Happiness Management» sobre las variables innovación, eficiencia, recursos humanos y resultado de las empresas. Por otro lado, que un acercamiento a este novedoso término enriquecerá sin lugar a duda su escasa literatura. 


\section{Referencias}

Ahn, N., \& Mochón, F. (2010). La felicidad de los españoles: factores explicativos. Revista de Economía Aplicada, 18(54), 5-31.

Ahumada-Tello, E., Ravina-Ripoll, R., Galiano-Coronil, A., \& Evans, R. D. (2018). Factors Affecting Corporate Happiness within Technology-Based Firms in Andalucia. En 2018 IEEE Technology and Engineering Management Conference (TEMSCON), pp.1-5. Illinois, Estados Unidos: IEEE. https://doi.org/10.1109/TEMSCON.2018.8488441

Nogueira, J., Varela, C., Batista, M., \& Weiler, M. (2017). Creativity and innovation for corporate happiness management. Brazilian Journal of Science and Technology, 4(1), 1-20. https://doi. org/10.1186/s40552-017-0038-7

Andersson, P. (2008). Happiness and health: Well-being among the self-employed. The Journal of Socio-Economics, 37(1), 213-236. https://psycnet.apa.org/doi/10.1016/j.socec.2007.03.003

Arunachalam, T. (2018). An Investigation on the Factor Structure of Hindi Version of Oxford Happiness Questionnaire (OHQ). Universitas Psychologica, 18(1), 1-11. https://doi.org/10.11144/ Javeriana.upsy18-1.ifsh

Bandura, R. P., \& Lyons, P. R. (2014). The art and science of job satisfaction. Human Resource Management International Digest, 22(7), 32-35. http://dx.doi.org/10.1108/hrmid-10-2014-0141

Baker, D., Greenberg, C., \& Hemingway, C. (2006). What Happy Companies Know. New Jersey, Estados Unidos: Pearson.

Benešová, A., \& Tupa, J. (2017). Requirements for education and qualification of people in Industry 4.0. Procedia Manufacturing, 11, 2195-2202. http://dx.doi.org/10.1016/j.promfg.2017.07.366

Blanchflower, D. G., \& Oswald A. J. (2011). International happiness: A new view on the measure or performance. The Academy of Management Perspectives, 25(1), 6-22. https://doi.org/10.5465/ amp.25.1.6

Blackman, D., O'Flynn, J., \& Mishra, D. P. (2010). Can strategic human resource management enable gross national happiness? International Journal of Commerce and Management, 20(3), 232245. https://doi.org/10.1108/10569211011076929

Cera, R. C. (2012). Nueva misión para la empresa competitiva: Happiness Management. Capital humano: revista para la integración y desarrollo de los recursos humanos, 25(265), 90-94.

Crespo, R. F., \& Mesurado, B. (2015). Happiness economics, eudaimonia and positive psychology: From happiness economics to flourishing economics. Journal of Happiness Studies, 16(4), 931-946. https://doi.org/10.1007/s10902-014-9541-4

Cuñado, J., \& De Gracia, F. P. (2012). Does education affect happiness? Evidence for Spain. Social indicators research, 108(1), 185-196. http://dx.doi.org/10.1007/s11205-011-9874-x

Diener, E. (2000) Subjective well-being: The science of happiness and a proposal for a national index. American Psychologist, 55(1), 34-43. http://dx.doi.org/10.1037/0003-066X.55.1.34

Dolan, P., \& Metcalfe, R. (2012). The relationship between innovation and subjective wellbeing. Research Policy, 41(8), 1489-1498. http://dx.doi.org/10.1016/j.respol.2012.04.001

Esteve, F. (2004). La economía de la felicidad: nuevos elementos para la crítica del liberalismo económico. En García, M.I. (Ed.), Filosofía y economía de nuestro tiempo: orden económico y cambio social (pp. 107-158). Madrid, España. Ministerio de Educación y Ciencia.

Ferrer i Carbonell, A. (2013). Happiness economics. SERIEs, 4(1), 35-60. http://dx.doi.org/10.1007/ s13209-012-0086-7

Fisher, C. D. (2010). Happiness at Work. International Journal of Management Reviews, 12(4), 384-412. https://doi.org/10.1111/j.1468-2370.2009.00270.x

Frey, B.S., \& Stutzer, A. (2010). Happines and economics: How the economy and institutions affect human well-being. New Jersey, Estados Unidos: Princeton University Press.

Frey, B. S., \& Stutzer, A. (2018). Economics of happiness. Basilea, Suiza: Springer International Publishing. 
Frijters, P., \& Beatton, T. (2012). The mystery of the U-shaped relationship between happiness and age. Journal of Economic Behavior $\mathcal{E}$ Organization, 82(2-3), 525-542. http://dx.doi.org/10.1016/j.jebo.2012.03.008

Graham, C., \& Pozuelo, J. R. (2017). Happiness, stress, and age: How the U curve varies across people and places. Journal of Population Economics, 30(1), 225-264. https://doi.org/10.1007/ s00148-016-0611-2

Helliwell, J., Layard, R., \& Sachs, J. (2017). World Happiness Report 2017. New York, Estados Unidos: Sustainable Development Solutions Network.

Hills, P., \& Argyle, M. (2002). The Oxford Happiness Questionnaire: a compact scale for the measurement of psychological well-being. Personality and Individual Differences, 33(7), pp. 10731082. http://dx.doi.org/10.1016/s0191-8869(01)00213-6

La Vanguardia (2018). Islas Fiji es el país más feliz del mundo, según encuesta de Gallup, 7 de enero. Recuperado de https://bit.ly/2K2jDWp

Kahneman, D., \& Krueger, A. B. (2006). Developments in the Measurement of Subjective Well-Being. Journal of Economic Perspectives, 20(1), 3-24. http://dx.doi.org/10.1257/089533006776526030

Lasi, H., Fettke, P., Kemper, H. G., Feld, T., \& Hoffmann, M. (2014). Industry 4.0. Business $\mathcal{E}$ information systems engineering, 6(4), pp. 239-242. http://dx.doi.org/10.1007/s11576-014-0424-4

Lu, L. (2000). Gender and conjugal differences in happiness. The Journal of social psychology, 140(1), 132-141. https://doi.org/10.1080/00224540009600451

Mackerron, G. (2012). Happiness economics from 35000 feet. Journal of Economic Surveys, 26(4), 705-735. http://dx.doi.org/10.1111/j.1467-6419.2010.00672.x

Maynard, A. D. (2015). Navigating the fourth industrial revolution. Nature Nanotechnology, 10(12), 1005-1006. http://dx.doi.org/10.1038/nnano.2015.286

Moreno, A., Velez, G., Ardanza, A., Barandiaran, I., de Infante, A., \& Chopitea, R. (2017). Virtualisation process of a sheet metal punching machine within the industry 4.0 vision. International Journal on Interactive Design and Manufacturing, 11(2), 365-373. https://doi.org/10.1007/ s12008-016-0319-2

Nelson, J. A. (2010). Getting past "rational man/emotional woman": comments on research programs in happiness economics and interpersonal relations. International Review of Economics, 57(2), 233-253. https://doi.org/10.1007/s12232-010-0096-3

Nogueira, J., Varela, C., Batista, M., \& Weiler, M. (2017). Creativity and innovation for corporate happiness management. Brazilian Journal of Science and Technology, 4(1), 1-20. https://doi. org/10.1186/s40552-017-0038-7

Núñez-Barriopedro, E., Ravina-Ripoll, R. \& Tobar-Pesantez, L. (2019). Creativity: An Intangible Capital Generating Competitive Quality in the Spanish Advertising Industry. Academy of Accounting and Financial Studies Journal, 23(2), 1-9. http://hdl.handle.net/10017/38016

Oishi, S. (2012). The psychological wealth of nations: Do happy people make a happy society? Massachusetts, Estados Unidos: Wiley-Blackwell.

ONU. (2011). Happiness should have greater role in development policy - UN Member States, 19 de Julio. Recuperado de https://bit.ly/2MAwgKf

Oswald, A. J., Proto, E., \& Sgroi, D. (2015). Happiness and Productivity. Journal of Labor Economics, 33(4), 789-822. https://doi.org/10.1086/681096

Pacek, A., \& Radcliff, B. (2008). Assessing the welfare state: The politics of happiness. Perspectives on Politics, 6(2),. 267-277. http://dx.doi.org/10.1017/S1537592708080602

Popkova, E. G., Ragulina, Y. V., \& Bogoviz, A. V. (Eds.) (2019). Industry 4.0: Industrial revolution of the 21st Century. Chan, Switzerland: Springer International Publishing.

Prause, M., \& Weigand, J. (2016). Industry 4.0 and object-oriented development: incremental and architectural change. Journal of Technological Management Innovation, 11(2), 104-110. http://dx.doi.org/10.4067/S0718-27242016000200010

Pryce-Jones, J., \& Lindsay, J. (2014). What happiness at work is and how to use it. Industrial and Commercial Training, 46(3), 130-134. https://doi.org/10.1108/ICT-10-2013-0072 
Ravina-Ripoll, R. (Coord). (2017). Entornos Creativos, Empleados Felices. Una ventaja competitiva en la gestión empresarial y territorial. Albacete, España: Uno Editorial.

Ravina-Ripoll, R., Villena-Manzanares, F., \& Gutiérrez-Montoya, G. A. (2017). Una aproximación teórica para mejorar los resultados de innovación en las empresas desde la perspectiva del "Happiness Management". RETOS. Revista de Ciencias de la Administración y Economía, 7(14), 113-129. http://dx.doi.org/10.17163/ret.n14.2017.06

Ravina-Ripoll, R., Núñez-Barriopedro, E., Galiano-Coronil A., \& Tobar-Pesantez, L. (2019). Towards A Happy, Creative and Social Higher Education Instituion: The Case of Non-Profit Marketing And Business Creation Subjects At The University Of Cadiz. Journal of Entrepreneurship Education, 22(1), 1-8. http://hdl.handle.net/10017/38017

Ravina-Ripoll, R., Tobar-Pesantez, L., \& Galiano-Coronil, A. (Coords). (2018). Claves para un desarrollo sostenible La creatividad y el happiness management como portafolio de la innovación tecnológica, empresarial y marketing social. Granada, España: Editorial Comares.

Ravina-Ripoll, R., Tobar-Pesantez, L., \& Marchena-Domínguez, J. (2019). A lighthouse for social wellbeing, creativity and sustainability: Happiness Management. Berna, Suiza: Peter Lang.

Ravina-Ripoll, R., Tobar-Pesantez, L., \& Núñez-Barriopedro, E. (Coords.) (2019). Happiness Management and Creativity in the XXI century: Intangible Capitals as a source of innovation, competitiveness and sustainable development. Granada, España: Editorial Comares.

Rego, A., Ribeiro, N., \& Cunha, M. P. (2010). Perceptions of organizational virtuousness and happiness as predictor of organizational citizenship behaviors. Journal of Bussiness Ethics, 93(2), 215-235. http://dx.doi.org/10.1007/s10551-009-0197-7

Rodríguez-Muñoz, A., \& Sanz-Vergel, A. I. (2013). Happiness and well-being at work: A special issue introduction. Revista de Psicología del Trabajo y de las Organizaciones, 29(3), 95-97. https:// doi.org/10.5093/tr2013a14

Rojas, M. (2009). Economía de la Felicidad: Hallazgos relevantes respecto al ingreso y el bienestar. El Trimestre Económico, 76(303), 537-573. http://dx.doi.org/10.20430/ete.v76i303.489

Rojas, M. (Ed.) (2016). Handbook of happiness research in Latin America. Dordrecht, Holanda: Springer.

Romero-Rodríguez, L.M., \& Castillo-Abdul, B. (2019). Comunicación para la motivación. Claves de la asertividad y del trabajo en equipo en las organizaciones. En R. Ravina-Ripoll, L.B. Tobar Pesántez y E. Nuñez Barriopedro (Coords.), Happiness Management and Creativity in the XXI Century. Intangible capitals as a source of innovation, competitiveness and sustainable development, (pp. 41-54). Granada, España: Editorial Comares.

Salas-Vallina, A., Alegre, J., \& Guerrero, R. F. (2018). Happiness at work in knowledge-intensive contexts: Opening the research agenda. European Research on Management and Business Economics, 24(3), 147-159. http://dx.doi.org/10.1016/j.iedeen.2018.05.003

Salas-Vallina, A., López-Cabrales, Á., Alegre, J., \& Fernández, R. (2017). On the road to happiness at work (HAW) Transformational leadership and organizational learning capability as drivers of HAW in a healthcare context. Personnel Review, 46(2), 314-338. https://doi. org/10.1108/PR-06-2015-0186

Sánchez-Vázquez, J. F. (2018). El significado vital en las organizaciones: aportaciones de la economía de la felicidad. CAURIENSIA, 13, 143-156. https://doi.org/10.17398/2340-4256.13.143

Saucedo-Martínez, J. A., Pérez-Lara, M., Marmolejo-Saucedo, J. A., Salais-Fierro, T. E., \& Vasant, P. (2018). Industry 4.0 framework for management and operations: a review. Journal of Ambient Intelligence and Humanized Computing, 9(3), 789-801. https://doi.org/10.1007/s12652017-0533-1

Seligman, M. (2016). Florecer: La nueva psicología positiva y la búsqueda del bienestar. México: Editorial Océano.

Smit, J., Kreutzer, S., Moeller, C., \& Carlberg, M. (2016). Industry 4.0 a study for the European Parliament. Recuperado de https://bit.ly/1QRykFi 
Sommer, L, (2015). Industrial Revolution-Industry 4.0: Are German manufacturing SMEs the first victims of this revolution? Journal of Industrial Engineering and Management, 8(5), 15121532. http://dx.doi.org/10.3926/jiem.1470

Valdivieso, J., \& Capella, A. (2009). Animales productivos. El papel económico de los animales no humanos y los retos morales que implica. Revista de Bioética y Derecho, 17, 50-57. https:// doi.org/10.1344/rbd2009.17.7773

Vázquez, C., \& Hervás, G. (2009). La ciencia del bienestar. Fundamentos de una psicología positiva. Madrid, España: Alianza Editorial.

Vázquez, S. (2012). La felicidad en el trabajo y en la vida. Madrid, España: Actualia Editorial.

Gaitán, I., Bretón, D., Choi, H., Mahecha, C., \& Arteaga, M. (2015). Todo es cuestión de actitud. Gestión de la felicidad. Harvard Deusto Business Review, 244, 6-17.

Villena-Manzanares, F., \& Galiano-Coronil, A. (2017). El desarrollo urbano sostenible y sus implicaciones para las empresas y los territorios. Revista de Estudios Empresariales, 1, 5-15. https://doi.org/10.17561/ree.v0i1.3185

Wacker, J. G. (1998). A definition of theory: research guidelines for different theory-building research methods in operations management. Journal of operations management, 16(4), 361-385. https://doi.org/10.1016/S0272-6963(98)00019-9

Wang, Y. D., \& Yang, C. (2016). How appealing are monetary rewards in the workplace? A study of ethical leadership, love of money, happiness, and turnover intention. Social Indicators Research, 129(3), 1277-1290. http://dx.doi.org/10.1007/s11205-015-1160-x

Will M. Bertrand, J., \& Fransoo, J. C. (2002). Operations management research methodologies using quantitative modeling. International Journal of Operations $\mathcal{E}$ Production Management, 22(2), 241-264. https://doi.org/10.1108/01443570210414338

Yuan, L. (2015). The Happier One Is, the More Creative One Becomes: An Investigation on Inspirational Positive Emotions from Both Subjective Well-Being and Satisfaction at Work. Psychology, 6(3), 201-209. http://dx.doi.org/10.4236/psych.2015.63019

Zelenski, J. M., Murphy, S. A., \& Jenkins, D. A. (2008). The happy-productive worker thesis revisited. Journal of Happiness Studies, 9(4), 521-537. http://dx.doi.org/10.1007/s10902-008-9087-4

Zweig, J.S. (2015). ¿Are women happier than man? Evidence from the Gallup World Poll. Journal of Happiness Studies, 16 (2), 515-541. http://dx.doi.org/10.1007/s10902-014-9521-8 